\title{
The application value of computed tomography and magnetic resonance imaging sinography/fistulography in evaluating chronic wounds: a prospective study
}

\author{
Hanqi Wang ${ }^{1 \#}$, Aobuliaximu Yakupu ${ }^{2 \#}$, Xian $\mathrm{Ma}^{2}$, Guilu Tao ${ }^{2}$, Di Zhang ${ }^{2}$, Zhihui Li ${ }^{3}$, Yalin Pan ${ }^{1}$, \\ Tongtong Chen ${ }^{1}$, Liuping Chen ${ }^{1}$, Haiying $\mathrm{Lv}^{1}$, Fuhua Yan ${ }^{1}$, Shuliang Lu${ }^{2}$, Yong Lu ${ }^{1}$ \\ ${ }^{1}$ Department of Radiology, Ruijin Hospital, School of Medicine, Shanghai Jiao Tong University, Shanghai, China; ${ }^{2}$ Wound Healing Center, Ruijin \\ Hospital, School of Medicine, Shanghai Jiao Tong University, Shanghai, China; ${ }^{3}$ Department of Radiology, Ruijin Hospital Luwan Branch, School of \\ Medicine, Shanghai Jiao Tong University, Shanghai, China \\ Contributions: (I) Conception and design: S Lu, Y Lu; (II) Administrative support: Y Lu; (III) Provision of study materials or patients: S Lu; (IV) \\ Collection and assembly of data: H Wang, A Yakupu; (V) Data analysis and interpretation: H Wang; (VI) Manuscript writing: All authors; (VII) Final \\ approval of manuscript: All authors. \\ \#These authors contributed equally to this manuscript. \\ Correspondence to: Shuliang Lu. Wound Healing Center, Ruijin Hospital, School of Medicine, Shanghai Jiao Tong University, No.197 Ruijin Er Road, \\ Shanghai 200025, China. Email: 13901738685@139.com; Yong Lu. Department of Radiology, Ruijin Hospital, School of Medicine, Shanghai Jiao \\ Tong University, No. 197 Ruijin Er Road, Shanghai 200025, China. Email: 18917762053@163.com.
}

Backgroundk Chronic wounds are a worldwide health problem, with traditional imaging techniques failing in their accurate evaluation. Therefore, an effective imaging evaluation method is needed for the diagnosis and treatment or chronic wounds. This study is to investigate the application value of computed tomography (CT) and magnetic resonance imaging (MRI) sinography/fistulography in assessing the morphology and deep features of chronic wounds.

Methods: We prospectively enrolled 43 chronic wounds patients who received both CT and MRI sinography/fistulography. The morphology and deep features of chronic wound on CT and MRI images were independently evaluated by 2 experienced radiologists. Kappa value and intraclass correlation coefficient (ICC) were calculated to evaluate the interobserver agreement and the consistency between CT and MRI sinography/fistulography in assessing the shape, number of branches, and involvement of body cavity and bones of chronic wounds.

Results: There were substantial to almost perfect interobserver agreements for both CT and MRI sinography/fistulography in evaluating the morphology and deep features of chronic wounds. The consistency between CT and MRI was almost perfect for the 2 readers in evaluating the shape (reader 1, kappa value $=1.000$; reader 2 , kappa value $=0.932)$ and the number of branches $[$ reader 1, ICC $=0.951(95 \%$ confidence interval: 0.909-0.973, $\mathrm{P}<0.001$ ); reader 2, ICC $=0.874$ (95\% confidence interval: $0.768-0.932$, $\mathrm{P}<0.001$ )], and substantial to almost perfect when evaluating the involvement of body cavity (reader 1 , kappa value $=0.728$; reader 2 , kappa value $=0.775)$ and bones (reader 1 , kappa value $=0.659$; reader 2 , kappa value $=0.860)$.

Conclusions: There was good interobserver agreement and consistency between CT and MRI sinography/ fistulography in evaluating the morphology and deep features of chronic wounds.

Keywords: Chronic wounds; computed tomography (CT); magnetic resonance imaging (MRI); sinography; fistulography

Submitted Jul 30, 2021. Accepted for publication Sep 18, 2021.

doi: 10.21037/apm-21-2342

View this article at: https://dx.doi.org/10.21037/apm-21-2342 


\section{Introduction}

Chronic wounds are wounds which fail to progress through the normal phases of wound healing or restore anatomic and functional integrity within 6 weeks $(1,2)$. It has been reported that chronic wounds affect $1-2 \%$ of the global population (3). The treatment and health care provision for chronic wounds have been a worldwide problem and cause increasing economic burden to patients and their governments $(4,5)$.

The majority of chronic wounds manifest as a tract or cavity with a small external opening, substantial depth, and sometimes multiple branches. They can also extend to the bone, body cavity, or another epithelial surface to form fistula (6). The complicated morphology and deep features of chronic wounds cause significant difficulties in diagnosis and treatment, therefore effective imaging evaluation is necessary. However, traditional imaging techniques such as X-ray sinography/fistulography, ultrasound, and laser surface scanning imaging are unable to accurately evaluate the morphology and deep features of chronic wounds (7).

Previous studies have reported the application of computed tomography (CT) and magnetic resonance imaging (MRI) in imaging evaluation of chronic wounds (8-12); CT scan relay high anatomic detail of chronic wounds and assess surrounding bone destruction (3), and MRI has high resolution and sensitivity to soft tissue without ionizing radiation. It is possible to combine CT and MRI with sinography/fistulography, which allows for visualization of internal features of the sinus/fistula by instilling contrast agent into the external opening (9). However, CT and MRI sinography/fistulography have not yet been widely applied and recommended as routine imaging techniques for chronic wounds, which is due to the complicated procedures, inevitable incompletely filling of contrast agent and requirement for highly integrated team of radiologists and surgeons. In this study, we investigated the application value of CT and MRI sinography/ fistulography in assessing the morphology and deep features of chronic wounds. We present the following article in accordance with the STROBE reporting checklist (available at https://dx.doi.org/10.21037/apm-21-2342).

\section{Methods}

\section{Study population}

All procedures performed in this study involving human participants were in accordance with the Declaration of Helsinki (as revised in 2013). This prospective study was approved by the institutional review board of Ruijin Hospital, Shanghai Jiao Tong University School of Medicine (No. 2018-27), and written informed consent was provided by each patient before undergoing CT and MRI scanning. Patients with chronic wounds (failure to heal in 6 weeks) were enrolled in Ruijin Hospital, Shanghai Jiao Tong University School of Medicine, China from January 2019 to April 2020. Exclusion criteria were standard safety contraindications to CT and MRI scanning. Besides, patients with insufficient contrast agent filling, incomplete imaging sequences, and lack of clinical data were excluded for images analysis (Figure 1). The final study population included a total of 43 patients [21 men $(48.8 \%)$ and 22 women $(51.2 \%)$, median age, 65 years (range, $18-94$ years)].

\section{CT and MRI Examination}

Before imaging examinations, surgeons performed endoscopy to clean chronic wounds to ensure that there was no foreign matter or pus affecting the imaging examinations. Participants took a supine, lateral, or prone position with the external opening upward and contrast agent was instilled into the external opening until it spilled over to fill the chronic wounds as full as possible. The instillation of contrast agent complied with the aseptic manipulation. Both CT and MRI scanning were performed on patients, and the time interval between CT and MRI scanning was less than a week. The CT scan was performed on a 256-slice CT scanner (uCT 760, United Imaging, Shanghai, China). The scan parameters were as follows: $120 \mathrm{kv}, 300 \mathrm{mAs}$, and section thickness of $1.0 \mathrm{~mm}$. The contrast agent for CT sinography/fistulography was $10 \%$ iomeprol (Iomeron, Bracco, Milan, Italy). The MRI scans were performed on a $1.5 \mathrm{~T}$ scanner (Magnetom Aera; Siemens Healthcare, Erlangen, Germany) using a 6-channel body phased array coil. The MR scanning sequences included axial T1-weighted (T1w), axial fatsaturated $\mathrm{T} 1 \mathrm{w}$, coronal or sagittal fat-saturated $\mathrm{T} 1 \mathrm{w}$, and axial fat-saturated T2-weighted (T2w). Parameters of the sequences are shown in Table 1. The contrast agent for MRI sinography/fistulography was $0.2 \mathrm{mmol} / \mathrm{L}$ gadopentetic acid dimeglumine (Magnevist, Bayer, Barmen, Germany). Multiplanar reconstruction (MPR) and maximum intensity projection (MIP) of CT images and axial T1w MR images were performed to achieve 3-dimensional (3D) visualization of chronic wounds. After scanning, surgeons cleaned the chronic wounds and changed the dressing to avoid infection 


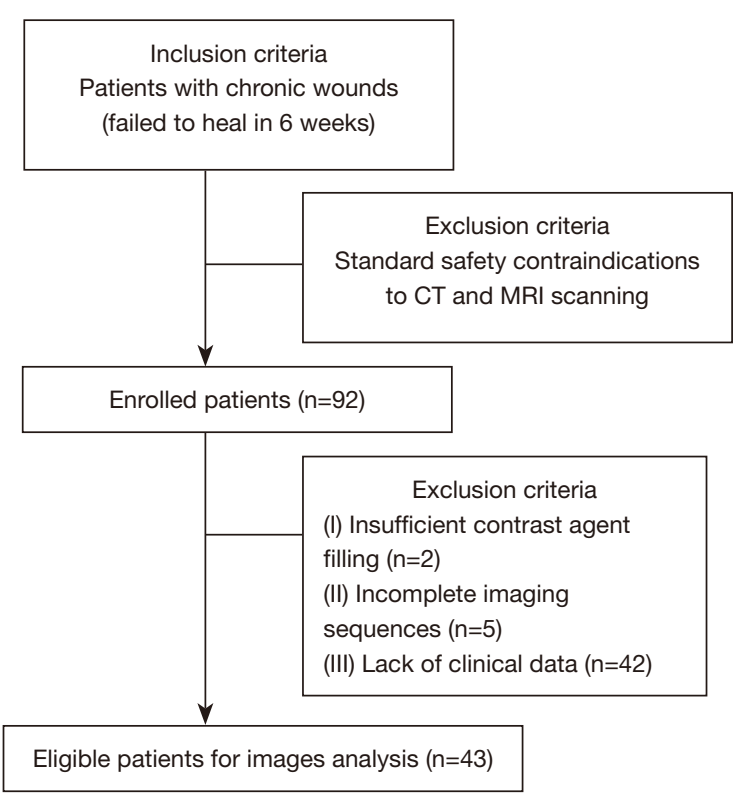

Figure 1 Flow chart illustrating the patient inclusion and exclusion criteria.

and the side effects of contrast agent.

\section{Images analysis}

For each anonymized participant, 2 imaging examinations were randomly allocated in 2 reading sessions by a referee not involved in the imaging analysis. A single examination (CT or MRI) per patient was presented in random order in each session. The images were individually reviewed by 2 trained radiologists with 7 years of experience (Z.L. and Y.P.), who were blinded to the clinical data in 2 sessions with a 4-week interval, and the following features were assessed: (I) shape of the chronic wound; (II) number of branches; (III) whether the chronic wound involved the body cavity; (IV) whether the chronic wound involved any bones. The shape of chronic wound was defined as a tract if the ratio of depth to maximum diameter was $>2$, or cavity if the ratio of depth to maximum diameter was $\leq 2$ (13).

\section{Statistical analysis}

Kappa value was calculated to evaluate the interobserver agreement and the consistency between CT and MRI sinography/fistulography in assessing the shape of chronic wounds and the involvement of body cavity and bones. Intraclass correlation coefficient (ICC) was calculated to evaluate the interobserver agreement and the consistency between CT and MRI sinography/fistulography in assessing the number of branches in chronic wounds. A kappa value or ICC value of $0.00-0.20$ indicated slight agreement; 0.21 0.40 , fair agreement; $0.41-0.60$, moderate agreement; $0.61-$ 0.80 , substantial agreement; 0.81 to less than 1.00 , almost perfect agreement; and 1.00, perfect agreement. Statistical significance was considered when $\mathrm{P}<0.05$. We used SPSS version 22.0 (IBM Corp., Armonk, NY, USA) to perform the statistical analysis.

\section{Results}

The demographic characteristics of participants are shown in Table 2. The chronic wounds were demonstrated as a contrast agent-filled tract or cavity with small bubbles $(65.1 \%, 28 / 43)$, surrounding fibrous connective tissue $(72.1 \%, 31 / 43)$, and effusion $(9.3 \%, 4 / 43)$.

The morphology of chronic wounds assessed by the 2 readers are shown in Table 3. There were substantial interobserver agreements for both CT and MRI sinography/fistulography. The consistency between CT and MRI in evaluating the shape of chronic wounds (reader 1 , kappa value $=1.000$; reader 2 , kappa value $=0.932)$ and the number of branches [reader 1, ICC $=0.951$ (95\% CI: 0.909-0.973, $\mathrm{P}<0.001$ ); reader 2 , ICC $=0.874$ (95\% CI: $0.768-0.932, \mathrm{P}<0.001)$ ] was almost perfect for the 2 readers.

The involvement of body cavity and bones in chronic wounds is shown in Table 4. The interobserver agreements for CT and MRI sinography/fistulography were substantial to almost perfect, which was same for the consistency between CT and MRI sinography/fistulography in evaluating the involvement of body cavity (reader 1, kappa value $=0.728$; reader 2 , kappa value $=0.775$ ) and bones (reader 1 , kappa value $=0.659$; reader 2 , kappa value $=0.860$ ) (Figure 2, Figure 3).

\section{Discussion}

Chronic wounds often form sinus/fistulas with complex deep structures, causing difficulties in their diagnosis and treatment. In this study, we found CT and MRI sinography/ fistulography had good interobserver agreements and consistency between each other in evaluating the shape, number of branches, and involvement of body cavity of chronic wounds. The results indicated that CT and MRI sinography/fistulography could be reliable imaging techniques for accurately assessing chronic wounds. 
Table 1 Parameters of the MRI protocol

\begin{tabular}{|c|c|c|c|c|c|c|c|}
\hline Parameters & $\mathrm{TR} / \mathrm{TE}(\mathrm{m} / \mathrm{s})$ & $\begin{array}{l}\text { Field of view } \\
\qquad\left(\mathrm{mm}^{2}\right)\end{array}$ & Matrix & $\begin{array}{l}\text { Slice thickness } \\
\qquad(\mathrm{mm})\end{array}$ & $\begin{array}{l}\text { Flip angle } \\
\text { (deg) }\end{array}$ & $\begin{array}{l}\text { No. of } \\
\text { slices }\end{array}$ & $\begin{array}{l}\text { Acquisition time } \\
\text { (min:s) }\end{array}$ \\
\hline \multicolumn{8}{|l|}{ Extremity } \\
\hline Axial fat-saturated $\mathrm{T} 1 \mathrm{w}$ & $700.00 / 10.00$ & 360 & $168 \times 320$ & 3.5 & 150 & 23 & $02: 00$ \\
\hline \multicolumn{8}{|l|}{ Thorax and abdomen } \\
\hline Axial T1w & $6.87 / 2.39$ & 360 & $175 \times 320$ & 5.0 & 15.0 & 56 & $00: 16$ \\
\hline Axial fat-saturated $\mathrm{T} 1 \mathrm{w}$ & $4.85 / 2.41$ & 380 & $195 \times 320$ & 3.0 & 10.0 & 64 & $00: 19$ \\
\hline Axial T1w & $827.00 / 21.00$ & 250 & $230 \times 256$ & 5.0 & 129 & 26 & $02: 30$ \\
\hline Axial fat-saturated $\mathrm{T} 1 \mathrm{w}$ & $827.00 / 21.00$ & 250 & $230 \times 256$ & 5.0 & 129 & 26 & 01:52 \\
\hline Axial fat-saturated T2w & $5,080.00 / 86.00$ & 250 & $272 \times 320$ & 6.0 & 180 & 24 & $02: 34$ \\
\hline Sagittal fat-saturated T1w & $827.00 / 21.00$ & 250 & $230 \times 256$ & 5.0 & 129 & 26 & $01: 56$ \\
\hline
\end{tabular}

MRI, magnetic resonance imaging; TR, repetition time; TE, echo time; T1w, T1-weighted; T2w, T2-weighted.

Table 2 Participant characteristics

\begin{tabular}{lc}
\hline Characteristic & $N(\%)$ or median [range] \\
\hline No. of included patients & $43(100.0)$ \\
Gender & $22(51.2)$ \\
Female & $21(48.8)$ \\
Male & $65[18-94]$ \\
Median age (years) & \\
Site of chronic wounds & $5(11.6)$ \\
Thorax & $17(39.5)$ \\
Abdomen & $2(4.7)$ \\
Lumbus & $13(30.2)$ \\
Hip & $2(4.7)$ \\
Upper extremity & $4(9.3)$ \\
Lower extremity & \\
Type of chronic wound & $40(93.0)$ \\
Sinus & $3(7.0)$ \\
Fistula & \\
\hline
\end{tabular}

Table 2 (continued)
Table 2 (continued)

\begin{tabular}{lc}
\hline Characteristic & $\mathrm{N}(\%)$ or median [range] \\
\hline Causes of chronic wound & $15(34.9)$ \\
Infection & $18(41.9)$ \\
Surgery & $1(2.3)$ \\
Malignancy & $9(20.9)$ \\
Pressure & \\
\hline
\end{tabular}

These imaging techniques also provide surgeons a more comprehensive look at the morphology and deep features of chronic wounds to make an appropriate treatment plan and efficacy evaluation.

Previous studies have reported the use of CT sinography/ fistulography in assessing the structural details of chronic wounds with sinus and fistula (9-12). Sun et al. found that CT fistulography and 3D image construction can clearly show the distribution, internal opening, and surrounding structure of congenital branchial cleft fistula and sinus (10). Thorpe et al. performed preoperative CT fistulography on 
Table 3 Morphology of chronic wounds

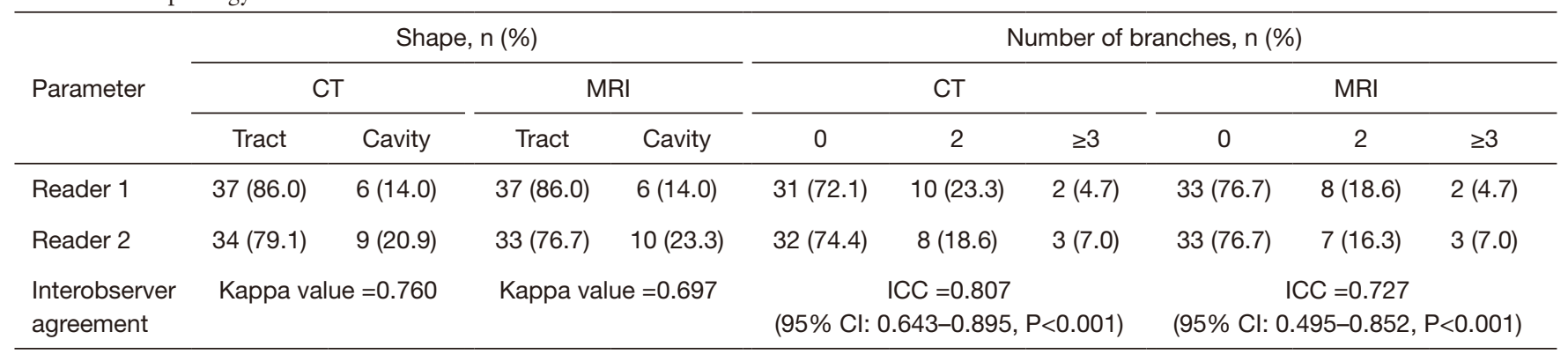

CT, computed tomography; MRI, magnetic resonance imaging; ICC, intraclass correlation coefficient; Cl, confidence interval.

Table 4 Involvement of body cavity and bones in chronic wounds

\begin{tabular}{|c|c|c|c|c|}
\hline Parameter & \multicolumn{2}{|c|}{ Body cavity, n (\%) } & \multicolumn{2}{|c|}{ Bones, n (\%) } \\
\hline Reader 1 & $7(16.3)$ & $6(14.0)$ & 7 (16.3) & $7(16.3)$ \\
\hline Reader 2 & $6(14.0)$ & $4(9.3)$ & $10(23.3)$ & $8(18.6)$ \\
\hline Interobserver agreement (kappa value) & 0.909 & 0.775 & 0.782 & 0.758 \\
\hline
\end{tabular}

$\mathrm{CT}$, computed tomography; MRI, magnetic resonance imaging.

a patient with branchial cleft sinus and found this technique facilitated excellent demonstration of the course and extent of the tract (11). The CT fistulography is also able to demonstrate the type of perianal fistula, its extent, whether it is simple or complex, and the site of internal opening and associated complications (9). However, CT sinography/ fistulography still has limitations such as low soft tissue resolution, high radiation dose, and inability to clearly show the morphology of sinus/fistula if the contrast agent is insufficiently filled.

The benefits of MRI include its sensitivity to soft tissue and ability to precisely define the extent of infection in wounds (14). Lee et al. found that MRI can show the location, internal and external openings of limited dorsal myeloschisis and congenital dermal sinus, and help to differentially diagnose them (15). Soker $e$ al. performed CT fistulography and MRI on perianal fistulae, and confirmed that both CT and MRI can clearly demonstrate the number of branches, with MRI showing more advantages in fistula classification, internal opening detection, and the identification of acute and chronic fistulas (12). Lane et al. found axial and sagittal MRI had high sensitivity and specificity in diagnosing dermal sinus of the lumbosacral spine in children (16). Besides, as 3D reconstruction of MR images help surgeons to observe chronic wounds from different angles, it has been shown to reveal the course, depth, and morphology more clearly (17). In our study, we combined MRI and sinography/fistulography to show the extent of sinus/fistula tracts and distinguish them from surrounding soft tissue more clearly. However, we also found CT has better performance for interobserver agreements than MRI, which may be due to that CT is more sensitive to contrast agent. Besides, CT has a higher ability in detecting some kinds of foreign matter such as small pieces of metal and plastic (18). Therefore, we should combine CT and MRI images in diagnosis of chronic wounds.

As for other imaging techniques used for the evaluation of chronic wounds, although conventional X-ray sinography/fistulography can show their shape and course, it is unable to clearly show the deep features and relationship with surrounding tissue, because of its low soft tissue resolution. Thus, it has a very limited role in diagnosis and treatment of chronic wounds. Ultrasound has been widely used in wound imaging because of its convenience, low cost, high resolution, and real-time response (3); however, it has limited penetration depth and does not easily reveal the deep features of chronic wounds. Besides, evaluation of narrow and tortuous sinus/fistula tract is susceptible to interference from surrounding muscle with similar echoes. 

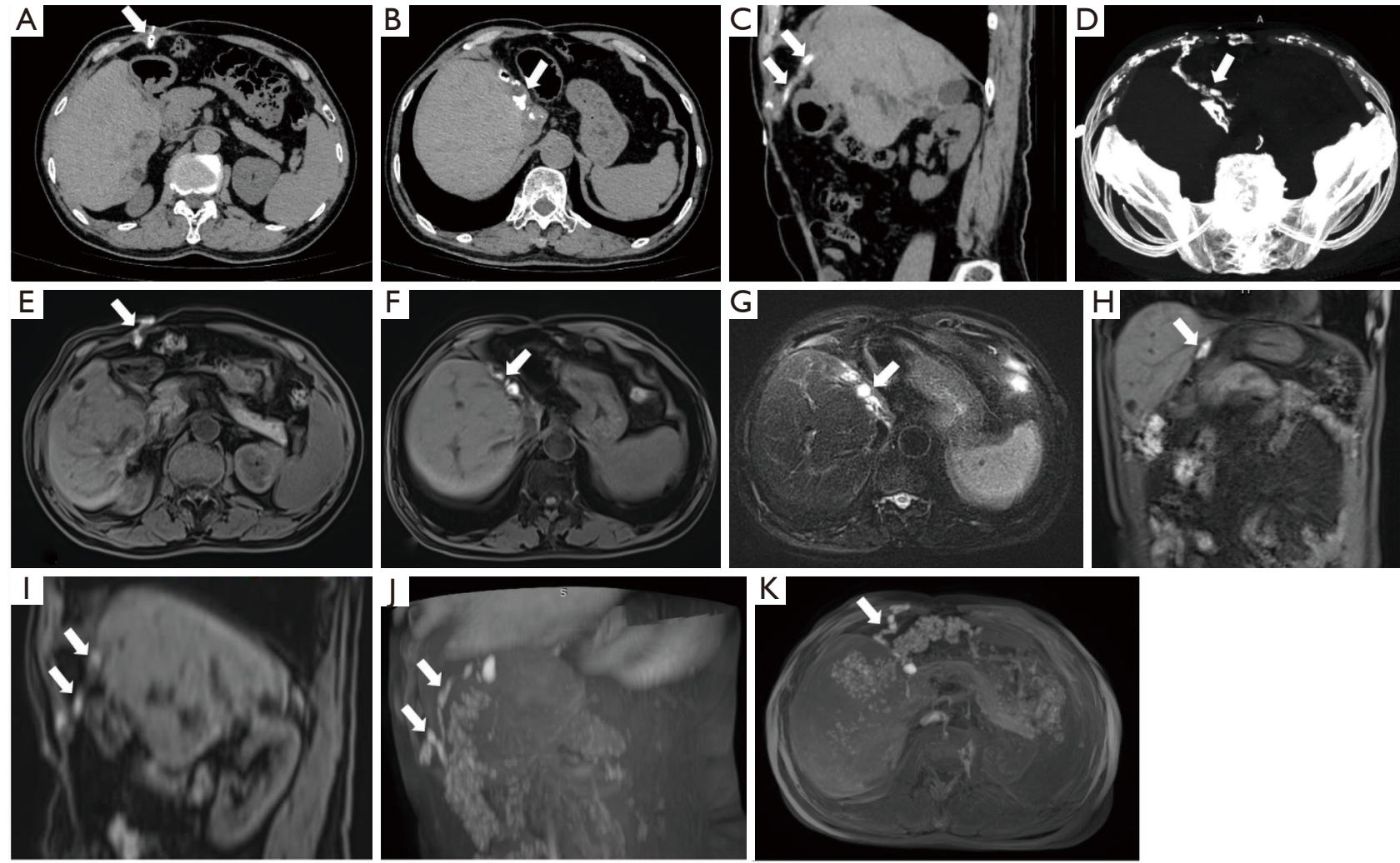

Figure 2 A 66-year-old man diagnosed with abdominal chronic wounds 2 years after partial hepatectomy. (A-D) CT images of sinography/ fistulography, MPR and MIP; (E-H) MR sinography/fistulography images of axial fat-saturated T1w, axial fat-saturated T2w and coronal fat-saturated T1w sequences; (I-K) MR images of MPR and MIP. The chronic wounds (white arrows) demonstrated as a contrast agent filled tortuous sinus tract with small bubbles, involving abdominal cavity and extending to liver. CT, computed tomography; MR, magnetic resonance; MIP, maximum intensity projection; MPR, multiplanar reconstruction.

Thus, ultrasound may miss branches in some cases and is unable to accurately assess the morphology and course of chronic wounds. Contrast-enhanced ultrasound may be able to lift its application restrictions, as it can show the morphology of chronic wounds better. But it has limited value for wounds with bone destruction, lung and digestive tract involvement.

There were some limitations to this study. First, it was a single-center prospective study with a limited sample size. Therefore, further validation of CT and MRI sinography/ fistulography is needed via larger cohort studies. Second, although we had taken measures to fill the wound as full as possible by contrast agent (i.e., the surgeons cleaned away foreign matter and pus before CT and MR scanning), the contrast agent may have still been insufficiently filled in some cases, especially those with narrow tract and multiple branches, which is thought to be one of the limitations of sinography/fistulography. Last, there was a lack of "gold standard" of chronic wound evaluation in the study. Endoscopy and conventional probe exploration often fail to reach the bottom of the wound or detect all the branches. Surgery helps to detect the deep features of chronic wounds; however, a few of the patients with chronic wounds received surgery, which may have destroyed the morphology of chronic wounds. It is therefore difficult to determine whether CT and MR images reflect the real morphology and deep features of chronic wounds. A combination of CT and MRI sinography/fistulography with endoscopy exploration may have the potential to become the gold standard of chronic wound evaluation. We need further study to evaluate its diagnostic value and compare it with other technologies such as ultrasound. 

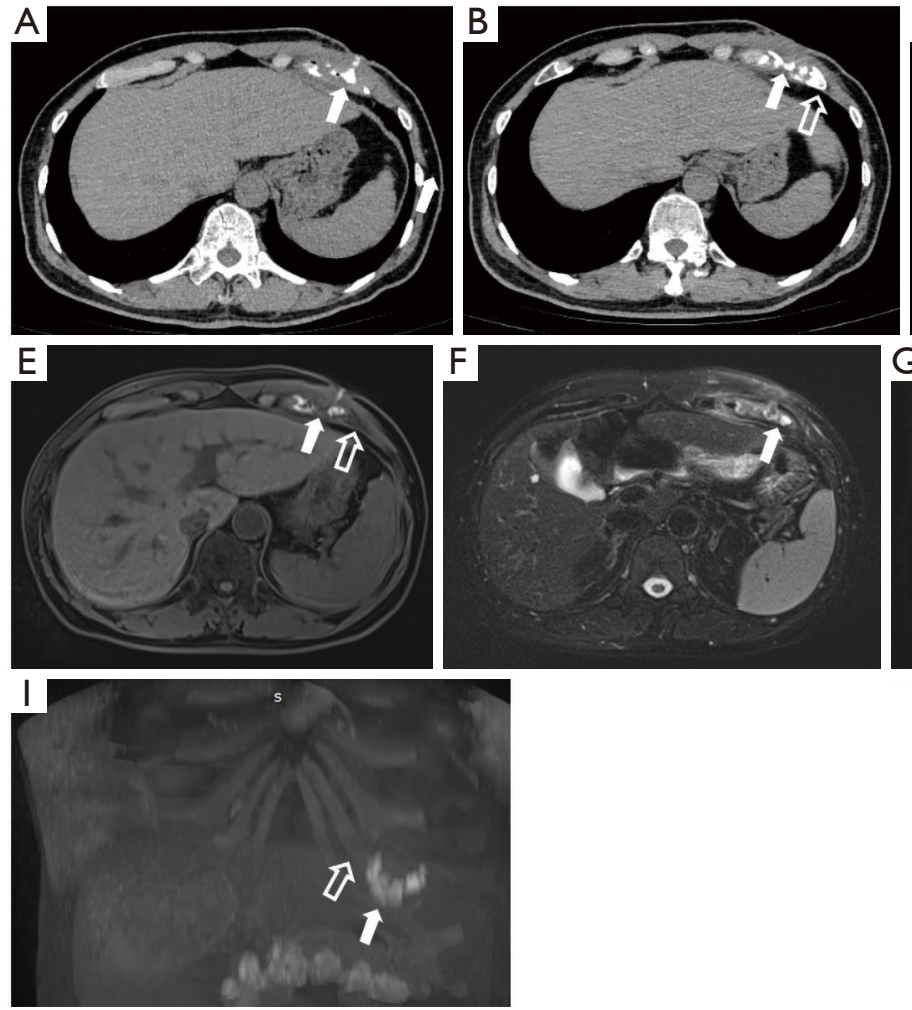
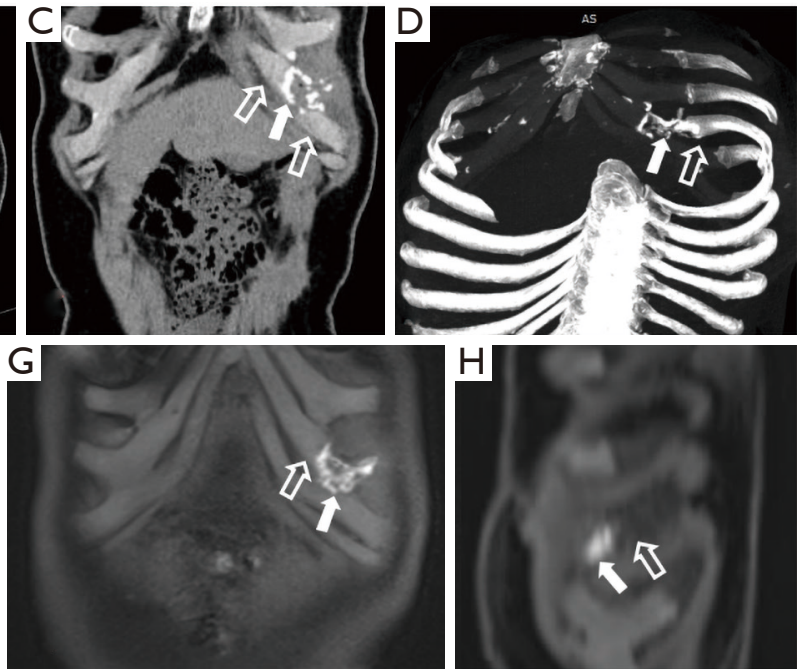

Figure 3 A 56-year-old man diagnosed with abdominal chronic wounds 10 months after abscess incision and drainage. (A-D) CT images of sinography/fistulography, MPR and MIP; (E-G) MR sinography/fistulography images of axial fat-saturated T1w, coronal fat-saturated T1w and axial fat-saturated T2w sequences; (H,I) MR images of MPR and MIP. The chronic wounds (white arrows) demonstrated as a contrast agent filled sinus cavity with small bubbles and surrounding soft tissue edema, involving left rib and costal cartilage (open arrows). CT, computed tomography; MR, magnetic resonance; MIP, maximum intensity projection; MPR, multiplanar reconstruction.

\section{Conclusions}

In conclusion, CT and MRI sinography/fistulography had good interobserver agreements and consistency between each other in evaluating the morphology and deep features of chronic wounds. Therefore, CT and MRI sinography/ fistulography could be reliable imaging techniques for chronic wounds and facilitate the evaluation of diagnosis, treatment, and healing.

\section{Acknowledgments}

Funding: This work was financially supported by the Action Plan of Major Diseases Prevention and Treatment (2017ZX01001-S12), the Project of Integrating Chinese and Western Medicine in General Hospital (ZHYYZXYJHZX-201901), and the Project of Shanghai Science and Technology Committee (21ZR1439800).

\section{Footnote}

Reporting Checklist: The authors have completed the STROBE reporting checklist. Available at https://dx.doi. org/10.21037/apm-21-2342

Data Sharing Statement: Available at https://dx.doi. org/10.21037/apm-21-2342

Conflicts of Interest: All authors have completed the ICMJE uniform disclosure form (available at https://dx.doi. org/10.21037/apm-21-2342). Dr. YL reports that this work was financially supported by the Action Plan of Major Diseases Prevention and Treatment (2017ZX01001-S12), the Project of Integrating Chinese and Western Medicine in General Hospital (ZHYY-ZXYJHZX-201901), and the Project of Shanghai Science and Technology Committee (21ZR1439800). The other authors have no conflicts of 
interest to declare.

Ethical Statement: The authors are accountable for all aspects of the work in ensuring that questions related to the accuracy or integrity of any part of the work are appropriately investigated and resolved. All procedures performed in this study involving human participants were in accordance with the Declaration of Helsinki (as revised in 2013). This prospective study was approved by the institutional review board of Ruijin Hospital, Shanghai Jiao Tong University School of Medicine (No. 2018-27), and written informed consent was provided by each patient.

Open Access Statement: This is an Open Access article distributed in accordance with the Creative Commons Attribution-NonCommercial-NoDerivs 4.0 International License (CC BY-NC-ND 4.0), which permits the noncommercial replication and distribution of the article with the strict proviso that no changes or edits are made and the original work is properly cited (including links to both the formal publication through the relevant DOI and the license). See: https://creativecommons.org/licenses/by-nc-nd/4.0/.

\section{References}

1. Bowers S, Franco E. Chronic Wounds: Evaluation and Management. Am Fam Physician 2020;101:159-66.

2. Greaves NS, Benatar B, Baguneid M, et al. Single-stage application of a novel decellularized dermis for treatmentresistant lower limb ulcers: positive outcomes assessed by SIAscopy, laser perfusion, and 3D imaging, with sequential timed histological analysis. Wound Repair Regen 2013;21:813-22.

3. Li S, Mohamedi AH, Senkowsky J, et al. Imaging in Chronic Wound Diagnostics. Adv Wound Care (New Rochelle) 2020;9:245-63.

4. Morton LM, Phillips TJ. Wound healing and treating wounds: Differential diagnosis and evaluation of chronic wounds. J Am Acad Dermatol 2016;74:589-605; quiz 605-6.

5. Frykberg RG, Banks J. Challenges in the Treatment of Chronic Wounds. Adv Wound Care (New Rochelle) 2015;4:560-82.

6. Schmid-Tannwald C, Agrawal G, Dahi F, et al. Diffusionweighted MRI: role in detecting abdominopelvic internal fistulas and sinus tracts. J Magn Reson Imaging 2012;35:125-31.

7. Jiang ZY, Liu MZ, Fu ZH, et al. Sinus Wounds Management. Adv Skin Wound Care 2018;31:1-4.

8. Han Z, Tai J, Gao J, et al. MRI in Children With Pyriform
Sinus Fistula. J Magn Reson Imaging. 2021 Jan;53(1):85-95.

9. Bhatt S, Jain BK, Singh VK. Multi Detector Computed Tomography Fistulography In Patients of Fistula-in-Ano: An Imaging Collage. Pol J Radiol 2017;82:516-23.

10. Sun Z, Fu K, Zhang Z, et al. Multidetector computerized tomographic fistulography in the evaluation of congenital branchial cleft fistulae and sinuses. Oral Surg Oral Med Oral Pathol Oral Radiol 2012;113:688-94.

11. Thorpe RK, Policeni B, Eigsti R, et al. CT Fistulography and Histopathologic Correlates for Surgical Treatment of Branchial Cleft Sinuses. Ear Nose Throat J 2020. [Epub ahead of print]. doi: 10.1177/0145561320933015.

12. Soker G, Gulek B, Yilmaz C, et al. The comparison of CT fistulography and MR imaging of perianal fistulae with surgical findings: a case-control study. Abdom Radiol (NY) 2016;41:1474-83

13. Ma X, Tang JJ, Wu MJ, et al. Diagnosis and treatment standard for treatment with endoscopy technique in chronic wounds with sinus tract (trial version). Zhonghua Shao Shang Za Zhi 2019;35:833-8.

14. Romanelli M, Miteva M, Romanelli P, et al. Use of diagnostics in wound management. Curr Opin Support Palliat Care 2013;7:106-10.

15. Lee SM, Cheon JE, Choi YH, et al. Limited Dorsal Myeloschisis and Congenital Dermal Sinus: Comparison of Clinical and MR Imaging Features. AJNR Am J Neuroradiol 2017;38:176-82.

16. Lane J, Dias M, Iantosca M, et al. Dermal Sinus Tract of Lumbosacral Spine in Children: Patterns on Magnetic Resonance Imaging and Scoring System. Cureus 2017;9:e1906.

17. Jia C, Li P, Cheng L, et al. Clinical application of surgical intervention model for repairing tuberculosis wound with sinus tract. Zhonghua Shao Shang Za Zhi 2016;32:326-30.

18. Javadrashid R, Fouladi DF, Golamian M, et al. Visibility of different foreign bodies in the maxillofacial region using plain radiography, CT, MRI and ultrasonography: an in vitro study. Dentomaxillofac Radiol 2015;44:20140229.

(English Language Editor: J. Jones)

Cite this article as: Wang H, Yakupu A, Ma X, Tao G, Zhang D, Li Z, Pan Y, Chen T, Chen L, Lv H, Yan F, Lu S, Lu Y. The application value of computed tomography and magnetic resonance imaging sinography/fistulography in evaluating chronic wounds: a prospective study. Ann Palliat Med 2021;10(10):10567-10574. doi: 10.21037/apm-21-2342 